\title{
The Relationship between Executive Functioning and Emotional Intelligence in Children with Autism Spectrum Disorder
}

\author{
Jennifer L. Reynolds, Alan J. Lincoln, Roya Iravani*, Varvara Toma, Shamayne Brown \\ California School of Professional Psychology, AIU, San Diego, USA \\ Email: *riravani@alliant.edu
}

How to cite this paper: Reynolds, J.L., Lincoln, A.J., Iravani, R., Toma, V. and Brown, S. (2018) The Relationship between Executive Functioning and Emotional Intelligence in Children with Autism Spectrum Disorder. Open Journal of Psychiatry, 8, 253-262.

https://doi.org/10.4236/ojpsych.2018.83022

Received: March 28, 2018

Accepted: July 10, 2018

Published: July 13, 2018

Copyright $(0) 2018$ by authors and Scientific Research Publishing Inc. This work is licensed under the Creative Commons Attribution International License (CC BY 4.0).

http://creativecommons.org/licenses/by/4.0/

\section{(c) (i) Open Access}

\begin{abstract}
Children with Autism Spectrum Disorder (ASD) demonstrate marked deficits in the ability to initiate, maintain and sustain meaningful social interaction. While the social-emotional deficits represent a core set of problems, persons with ASD also demonstrate significant problems in initiating, sustaining and maintaining appropriate goal directed behaviors. Emotional Intelligence (EI) is a construct that has been successfully applied to a range of skills that allow for the prediction of competent human social behavior. Executive Functions (EF) refer to constructs involving cognitive abilities necessary for initiating, sustaining and maintaining purposeful goal-oriented behavior. While both children and adults with ASD have previously shown to have atypical patterns of EF skills, little is known about EI in either children or adults with ASD. Moreover, there is no study examining the relationship between EI and EF that has been reported in individuals with ASD. The current study examined the relationship between EF and EI in children with ASD. Twenty children with ASD were compared to twenty neurotypical children on self-report and clinical assessments of EI and EF. Although the relationship between EF and EI was not statistically significant, results showed that children with ASD have deficits in interpersonal skills, intrapersonal skills and overall EI when compared to their neurotypical peers. These results suggest that EF and EI are relatively independent domains of development that show compromise in persons with ASD and each may be necessary to support typical socially directed behaviors.
\end{abstract}

\section{Keywords}

Executive Functioning, Emotional Intelligence, Autism Spectrum Disorder 


\section{Introduction}

Social interaction deficiencies and communication problems are among the most pronounced in children with ASD (Baron-Cohen, Leslie \& Frith, 1995) [1] and often the first area of intervention for practitioners. Children with ASD often play alone, have difficulty in initiating and sustaining play with other children, and show limitations in social imagination (Seitz, Travis \& Sigman, 2005) [2]. Moreover, their capacity is reduced for recognizing and responding to the emotional states of others.

EI refers to the ability to assess and engage the emotions of one's self, of others and of groups (Mayer, 1997) [3]. The somatic marker hypothesis suggests that deficits in the emotional signaling functional system (somatic states) lead to poor judgment in decision-making, especially in the personal and social realms (Bar-On, Tranel, Denburg \& Bechara, 2003) [4]. This system is necessary to facilitate social interaction, imaginative activities, and the use of emotions for social communication and has been hypothesized to comprise an area of social abilities known as emotional intelligence (EI).

Individuals with ASD show social deficits that would suggest impairment in two general areas of EI: intrapersonal and interpersonal skills. Intrapersonally, children with ASD show a deficit in the ability to discriminate among emotions, overtly label emotions, utilize emotions to guide their own behaviors and effectively control affective range and intensity (Begeer, Rieffe, Terwogt, Meerum \& Stockmann, 2006) [5]. Interpersonally, children with ASD show difficulties in correctly decoding other people's feelings, intentions, and motivations, correctly recognizing personal characteristics in others (e.g., age, gender, and ethnicity) and influencing others to behave in desired ways (Dalrymple, 1992) [6].

Neurobiological and neuropsychological evidence suggest that the significant range of social deficiencies observed in individuals with ASD may be more effectively viewed as the abnormal development of a distinct functional brain system (McAlonan, Cheung, Suckling, Lam, Tai, Yip, Declan and Siew, 2005) [7]. One such system includes to so-called executive functions that are believed to be subserved by the frontal cortex and appear to be responsible to help regulate the capacity for initiating, sustaining and maintaining goal directed behaviors. Early work by Pennington and Ozonoff (1996) [8] and others (Lopez, Lincoln, Ozonoff, \& Lai, 2005) [9] showed that persons with ASD show atypical patterns of EF regulation and suggested that impairments or the atypical organization of such functions could account for impaired social and behavioral functioning in persons with ASD.

Despite the fact that EF has been studied extensively in individuals with ASD, there is still limited research in the area of EI and individuals with ASD and even sparser research examining the correlational link between EF and EI. Currently, neurobiological and neuropsychological research supports the idea that $\mathrm{EF}$ and EI are linked by way of subcortical structures [7]. Furthermore, [4] found that when compared to controls, patients with lesions in the neural circuitry post- 
ulated to be involved in EF and EI, displayed low EF and EI scores as assessed by standardized testing. Unfortunately, the research examining the neural circuitry has been limited to non-ASD individuals, suggesting a need for further research in this area with a developmentally-delayed subpopulation.

The current study was designed to evaluate whether children with ASD would achieve lower scores on measures that reflect the following executive functions: cognitive flexibility, inhibition, initiation of problem solving, concept formation and spatial planning. Emotional Intelligence was also predicted to differ between autistic participants and control participants. Finally, it was predicted that the relationship between EF and EI would differ between children with ASD and neuro typical control children.

\section{Methods}

\subsection{Participants}

Children in the ASD group $(N=20)$ between the ages of 8 and 16 had a verified current diagnosis of ASD (DSM-V, 2013) [10] or were administered the Autism Diagnostic Observational Schedule (ADOS: Lord, 1989) [11] to confirm the diagnosis. The Gilliam Autism Rating Scale-2 (GARS-2; Gilliam, 2005) [12] was used to assess for ASD symptoms. The inclusion criteria for children with ASD included: 1) being between ages of 8 - 16,2) having an IQ of 85 or greater as measured by the Wechsler Intelligence Scale for Children Fourth Edition (WISC-IV; Wechsler, et al., 2003) [13], and 3) meeting criteria for ASD on the ADOS and GARS. Exclusion criteria included parental report of the following: 1) known neurodevelopmental or genetic disorder, 2) prior traumatic brain injury or 3) a coexisting mental disorder.

The control group consisted of neurotypical children (NC) $(N=20)$, between the ages of 8 and 16. The inclusion criteria for the control group consisted of: 1) being between the ages of 8 - 16 and 2) having an IQ as determined by the WISC-IV of 85 or greater. Exclusion criteria were the same as the ASD group. Sample size was determined by conducting a power analysis using the effect size from Gilotty et al. (2002) [14]. This study was approved by the Institutional Revie board of Alliant International University and was conducted between 2009-2012.

\subsection{Measures}

Demographic Questionnaire (e.g. age, gender) was administered to all participants. ASD diagnosis was confirmed using ADOS, a semi-structured instrument used to assess for ASD; and GARS, a structured instrument used to additionally asses for ASD. IQ was assessed using WISC-IV, a cognitive assessment that gives a comprehensive picture of child's intellectual ability. EF was measured using the Delis Kaplan Executive Function System (DKEFS; Delis, Kaplan \&Kramer, 2001) [15], a set of neuropsychological tests used to measure verbal and non-verbal EF for both children and adults. EI was measured using the BarOn Emotional Quo- 
tient-Inventory-Youth Version (BAR-ON EQi:Y-V; Bar-On \& Parker, 2000) [16], a self-report focused on EI and social functioning.

\subsection{Procedures}

The first session was conducted with parents to complete informed consent, evaluate inclusionary and exclusionary criteria for each participant, demographic questionnaire and GARS. In a separate three hour session the child was administered the ADOS, D-KEFS, WISC-IV and the BAR-ON EQi:Y-V. The ADOS and WISC-IV were only given if it had not been given within the past year and/or scores from the most recent evaluation were unavailable.

\subsection{Statistical Analysis}

A one way ANOVA was used to determine between group differences for the $\mathrm{AD}$ and NC groups on tests of D-KEFS Trail Making, D-KEFS Color Word Inference, and D-KEFSCard Sort. Furthermore correlations were conducted for the AD group between the DKEFS Free Sort Score, DKEFS Trail Making Combined score, DKEFS Verbal Fluency Combined score, DKEFS Color Word Inference Combined score, and the Emotional Intelligence Interpersonal, Intrapersonal, and Adaptability scores. Correlations were also runo DKEFS subtests of cognitive flexibility and planning and BarOnEQi scales of emotional intelligence.

\section{Results}

\section{Descriptive Statistics}

Children with ASD $(N=20)$ and neurotypical children $(N=20)$, all received intellectual testing, EF testing, and completed an EI self-report questionnaire. All of the control and experimental participants met the inclusionary and exclusionary criteria with the exception of one participant with ASD whose IQ score did not meet the inclusionary criteria.

There were no significant differences on age $t(1,37)=0.291, p=0.533$ (Masd $=11.1, S D$ asd $=2.71, M \mathrm{nc}=10.8, S D \mathrm{nc}=2.81)$ or gender, $X^{2}(1, \mathrm{~N}=39)=9.9, p$ $=0.353$. The ethnicity of the ASD group consisted 60\% Caucasian, 15\% Hispanic, 20\% Other and 5\% Decline to State. The ethnicity of the NC group consisted of 55\% Caucasian, 35\% Hispanic and 5\% Other. The intellectual abilities of both the ASD group and the NC group were compared (Table 1).

A one-way ANOVA of the D-KEFS Trail Making Combined Scaled scores was conducted. There was a significant difference between the AD group and the NC group $\mathrm{F}(1,38)=4.22, \mathrm{p}=0.047$. effect size for partial $\mathrm{n}^{2}=0.896$. The $\mathrm{AD}$ group displayed significantly lower scores on the letter number switching variable than did the NC group, suggesting a planning deficit and cognitive flexibility deficit and confirming the hypothesis H1. A one-way ANOVA with the D-KEFS Design Fluency Combined scaled scores was conducted and was not significant $F(1$, $18)=3.04, p=0.756$. However, since homogeneity of variance was violated, a 
Table 1. Descriptive statistics of WISC-IV scores for the AD and the NC groups.

\begin{tabular}{|c|c|c|c|c|c|c|}
\hline \multirow{3}{*}{ WISC-IV Subtest } & \multicolumn{6}{|c|}{ Groups } \\
\hline & \multicolumn{2}{|c|}{ AD Group } & \multicolumn{2}{|c|}{ NC Group } & \multicolumn{2}{|c|}{$\begin{array}{l}\text { Difference between } \\
\text { groups }\end{array}$} \\
\hline & M & $\mathrm{SD}$ & M & SD & t-test & $\mathrm{p}$ value \\
\hline $\begin{array}{l}\text { Verbal Comprehension } \\
\text { Index }\end{array}$ & 101.75 & 18.90 & 108.26 & 11.58 & 1.30 & $0.201^{\mathrm{c}}$ \\
\hline $\begin{array}{l}\text { Perceptual Reasoning } \\
\text { Index }\end{array}$ & 107.35 & 15.12 & 108.58 & 13.22 & 0.270 & 0.789 \\
\hline Working Memeroy Index ${ }^{a}$ & 105.60 & 16.21 & 104.95 & 14.00 & -0.134 & 0.894 \\
\hline Processing Speed Index ${ }^{\mathrm{a}}$ & 91.20 & 13.20 & 87.95 & 14.52 & -0.733 & 0.468 \\
\hline Full Scale Index ${ }^{\mathrm{a}}$ & 103.10 & 15.02 & 104.26 & 8.96 & 0.295 & $0.770^{\mathrm{c}}$ \\
\hline Block Design $^{\mathrm{b}}$ & 12.15 & 2.64 & 11.37 & 2.60 & -0.929 & 0.359 \\
\hline Similarities $^{\mathrm{b}}$ & 12.10 & 3.07 & 12.42 & 2.52 & 0.355 & 0.724 \\
\hline Digit Span ${ }^{\mathrm{b}}$ & 12.60 & 3.51 & 12.21 & 3.20 & -0.361 & 0.720 \\
\hline Picture Concepts ${ }^{\mathrm{b}}$ & 9.45 & 3.72 & 11.16 & 3.27 & 1.59 & 0.137 \\
\hline Coding ${ }^{\mathrm{b}}$ & 8.30 & 3.52 & 6.84 & 2.73 & -1.43 & 0.159 \\
\hline Vocabulary $^{\mathrm{b}}$ & 9.20 & 4.20 & 11.05 & 2.63 & 1.65 & $0.107^{\mathrm{c}}$ \\
\hline $\begin{array}{l}\text { Letter-Number } \\
\text { Sequencing }\end{array}$ & 9.45 & 2.60 & 9.79 & 2.70 & 0.400 & 0.692 \\
\hline Matrix Reasoning ${ }^{\mathrm{b}}$ & 11.50 & 2.80 & 11.63 & 3.23 & 0.136 & 0.893 \\
\hline Comprehension $^{\mathrm{b}}$ & 7.65 & 3.54 & 11.26 & 2.28 & $3.76^{*}$ & 0.001 \\
\hline Symbol Search ${ }^{\mathrm{b}}$ & 8.20 & 2.66 & 8.79 & 2.78 & 0.676 & 0.503 \\
\hline
\end{tabular}

${ }^{a}$ denotes the measure has $\mathrm{M}=100, \mathrm{SD}=15$, ${ }^{\mathrm{b}}$ denotes the measure has $\mathrm{M}=10, \mathrm{SD}=3$, 'denotes Levene's test for equality of variance was significant.

nonparametric Mann-Whitney $U$ test was conducted. The Mann Whitney $\mathrm{U}=$ 180.50, $\mathrm{Z}=-0.269$, exact significance $=0.792$ was not significant. A one-way ANOVA for the D-KEFS Tower Test Total Achievement scaled score was conducted. There was a significant difference between the $\mathrm{AD}$ group and the $\mathrm{NC}$ group $\mathrm{F}(1,38)=5.928, \mathrm{p}=0.020$. Effect size for partial $\mathrm{n}^{2}=0.490$. Additionally, the $\mathrm{AD}$ group displayed higher scores on total rule violation thus indicative of problems with cognitive flexibility, inhibition and initiation of problem solving.

There was a significant difference between the $\mathrm{AD}$ group and the NC group on D-KEFS Color Word Inference Total Error scaled scores F $(1,38)=4.693, \mathrm{p}=$ 0.037. Effect size for partial $\mathrm{n}^{2}=0.891$.

There was a marginal significant difference between the $\mathrm{AD}$ group and the $\mathrm{NC}$ group for the D-KEFS Card Sort Combined scaled scores, $\mathrm{F}(1,38)=1.920, \mathrm{p}=$ 0.082. Effect size for partial $\mathrm{n}^{2}=0.439$.

The AD group received lower scores on the Intrapersonal Scale, Interpersonal Scale, and EQ Total Scale. The groups differed slightly on the Adaptability Scale and General Mood Scale with the NC group doing slightly worse on both. 
Descriptive statistics of Scales of the BarOn Emotional Quotient Inventory: Youth Version.

Correlations between DKEFS subtests for Cognitive Flexibility and Planning and BarOn EQi Scales of Emotional Intelligence

Upon initial examination of the bivariate scatterplots, it was evident that there was a linear relationship between the planning variable, cognitive flexibility variable, and initiation variable and the criterion. No significant correlations emerged. Neither planning, inhibition nor cognitive flexibility related to the criterion (overall emotional intelligence). Gender and age did not correlate with the criterion in its continuous form. Therefore, it was not necessary to use these variables as covariates. The multicollinearity among predictors was within acceptable range for the regression. The histograms revealed normal distribution and the assumptions of linearity and homoscedasticity were met. The three predictors accounted for $14 \%$ of the variance in emotional intelligence $(\mathrm{F}(3,35)=1.90$, $\mathrm{p}<0.147)$. The unstandardized beta weight was marginally significant for DKEFS Sort $(B=0.751, p<0.069)$ and non-significant for DKEFS Design Fluency $(B=0.074, p<0.839)$ and non-significant for DKEFS Tower $(B=-0.097$, $p$ $<0.829)$.

\section{Discussion}

It has been long understood that the social impairment in individuals with ASD is often severe and a hallmark sign of the disorder. The primary purpose of this study was to examine the relationship between executive functions and emotional intelligence in children with ASD. Findings from this study replicated previous findings related to executive dysfunction in children with ASD and produced findings related to emotional intelligence in a developmentally delayed population.

The analysis conducted replicated previous findings showing that children with ASD display deficiencies in cognitive flexibility and planning. In the current study, autistic individuals performed significantly worse on tasks that required cognitive flexibility and planning. The deficits were most obvious on the trail making task; a task that requires set shifting ability and planning skills. Moreover, there was a significant difference found on the color word inference task between the AD and the NC group. Specifically, it was found that on the switching tasks, autistic individuals performed significantly worse than the NC group. These findings support previous research findings that children with ASD have difficulty spontaneously restructuring their knowledge in adaptive response to radically changing situational demands.

It was also assumed that children with ASD would perform worse on the DKEFS Design Fluency task; a task that requires individuals to follow rule set to make designs. The results did not produce significant findings on the contrast measure, a measure that examines the difference between following simple instructions to connect dots and instructions to switch back and forth between 
connecting filled and empty dots. Upon exploratory analysis however, it was found that the $\mathrm{AD}$ group took more time to complete the designs and made more mistakes, which suggest a planning deficiency. This also suggests that processing speed may be an impediment with regards to their performance. Moreover, the higher number of mistakes made by the AD group on this specific shows deficits in planning and perseveration are linked to the frontal and prefrontal areas in the brain and are often observed in children with ASD.

The current study supported the previous research by showing that the AD group performed significantly worse than the NC group on the DKEFS Tower Test. Moreover, contrast measures showed that the AD group had more rule violations and took more time to complete towers. Additionally, the DKEFS Card Sort test resulted in the $\mathrm{AD}$ group generating significantly fewer sorts than the $\mathrm{NC}$ group. Contrast measures further showed that the $\mathrm{AD}$ group took more time to recognize and generate sorts, thus supporting difficulty with planning and spontaneous cognitive generation.

Furthermore, the current study looked at emotional intelligence, as a new emerging construct, and also what it looked like in a developmentally delayed youth population when compared to a normally developing youth population. Further, with an abundance of research supporting a somatic marker hypothesis (cortical under-connectivity), the study was designed to examine the relationship between executive functioning and emotional intelligence. Following emotional intelligence is a branch system of traits that includes intrapersonal and interpersonal intelligence, stress management and adaptability.

In the current study emotional intelligence would be shown to differ between autistic and non-autistic individuals. The findings suggested significant difference on the interpersonal scale between the two groups. Interestingly, the results indicated that the NC group obtained lower scores on the adaptability and stress management scales. The current study thought that cognitive flexibility and planning scores (main measures of executive functioning) would be significantly correlated to emotional intelligence scores. However, it was found that no significant relationship exists between the global indices of each of the variables. Additionally, when specific skill sets of emotional intelligence and individual components of executive functioning were analyzed, no significant correlations emerged.

Additionally, the study examined the ability of cognitive flexibility, inhibition and planning (executive functions) to predict emotional intelligence. The results showed that there were no significant correlations and neither planning, inhibition nor cognitive flexibility related to emotional intelligence.

The results of this study supported previous research findings that children with ASD display marked deficits in executive functioning when compared to non-autistic children [9]. More specifically, the results showed that children with ASD have specific impairments with the ability to spontaneously restructure their knowledge, in many ways, in adaptive response to radically changing situational demands. They showed deficits in the ability to initiate and stop actions, 
to monitor and change behavior as needed and to plan future behavior when faced with novel tasks and situations. Moreover, the results showed that the $\mathrm{AD}$ children did not have the ability to effectively anticipate outcomes and adapt to changing situations, form concepts and think abstractly. These results imply that it is important for autistic children to strengthen their executive functioning skills by way of learning, and practicing, skill sets such as goal setting, problem identification, plan development and execution, attention, working memory, and self-monitoring.

The current study also showed that autistic children have difficulty with emotional intelligence traits. Specifically, when compared to non-autistic children, the $\mathrm{AD}$ group showed marked deficiencies with interpersonal intelligence; the ability to communicate their emotions verbally and non-verbally, viewing situations from different perspectives, the ability to create positive relationships with others and adequate conflict resolution skills. Additionally, findings showed that the AD children had difficulty with intrapersonal skills; distinguishing themselves from the environment and understanding their own emotions, motivations and behaviors. The emotional intelligence deficiencies suggest that it is important for children with ASD to be involved in social and emotional literacy programs. Furthermore, since it is strongly supported in research [4] that emotional intelligence is a multibranched construct, it is important to examine individual deficiencies within each trait to improve upon those specific skills.

The results from this study also suggest the possibility that the component deficits in both emotional intelligence and executive functioning may be due to additional biological or environmental processes that no single construct can account for. Due to the fact that ASD varies so widely in terms of symptom manifestations, it is imperative that individual differences within each construct (executive functioning, emotional intelligence) be examined.

\section{Limitations}

Despite the fact that this study contributed to the literature, there were limitations that must be noted. There was not an opportunity for random assignment of subjects, so the quasi experimental design makes it impossible to say that the independent variables affected the dependent variable.

Another limitation involves the conceptualization, definition and assessment of the constructs themselves. Because of the wide variability of discrete skills involved in executive functioning, it is difficult to directly measure every discrete part of each skill set.

Another limitation is associated with BarOn EQi:YV assessment used in this study. While this test is considered to be valid and reliable, the test cannot measure a person's innate emotional intelligence while excluding environmental factors. Further, the test is too subjective, the answers depend on the examinee's culture, beliefs and experiences. Moreover, because it is a self-report measure, it can contain bias and variable error. In addition, autistic individuals may not 
have the self-awareness and insight that it takes to answer the questions honestly and as a result, the results that were obtained may be more a measure of their perceived emotional intelligence rather than their actual skill or capacity. Future research could supplement the BarOn EQi:YV by utilizing other forms of emotional intelligence assessment tools; such as facial recognition tasks or theory of mind tasks.

A further limitation was the restriction of diagnosis within the $\mathrm{AD}$ group. Only 3 of the 18 children that were assessed were diagnosed with low functioning ASD; the remaining children were all diagnosed with high functioning ASD. The phenotypical differences between children who are low and high on the continuum is markedly different as are their symptoms; therefore, it would be important for future research to look at the relationship between emotional intelligence and executive functioning in children all along the continuum.

Finally, another limitation of the study was the average sample size from which the data was derived. Due to the IQ exclusionary criteria, the recruitment efforts and participation reward, the study group size was limited. As a result of the average sample size, the present study may have limited statistical power to detect significant effects.

\section{Conclusion}

The present study supported previous research that children with ASD have executive functioning deficits; specifically, in the areas of planning, cognitive flexibility and inhibition. Although the current research did not support a predictive relationship between executive functions and emotional intelligence, it did add to the field of research in emotional intelligence. The findings suggest that even with a loosely operationalized construct, children with ASD, when compared to their normal peers, show deficits in their ability to relate to others, initiate and maintain interpersonal relationships, discriminate among their own emotions and the emotions of others and utilize emotions to guide their own behaviors. This study did support the current debate about utilizing self-report as the only measure to assess emotional intelligence.

\section{References}

[1] Baron-Cohen, S., Leslie, A.M. and Frith, U. (1995) Does the Autistic Child Have a "Theory of Mind"? Cognition, 21, 37-46. https://doi.org/10.1016/0010-0277(85)90022-8

[2] Seitz, J., Travis, L. and Sigman, M. (2005) Social Deficits and Interpersonal Relationships in ASD and Mental Retardation. Developmental Disabilities Research Review Special Issue: $A S D, 42,657-672$.

[3] Mayer, J.D. and Salovey, P. (1997) What Is Emotional Intelligence: Implications for Educators. In: Salovey P. and Sluyterr, D., Eds., Emotional Development and Emotional Intelligence: Educational Implications, Harper Collins, New York, 3-34.

[4] Bar-On, R., Tranel, D., Denberg, N.L. and Bechara, A. (2003) Exploring the Neurological Substrate of Emotional and Social Intelligence. Journal of Neurology, 126, 
1790-1800.

[5] Begeer, S., Rieffe, C., Terwogt, M.M. and Stockmann, L. (2006) Attention to Facial Emotion Expressions in Children with ASD. ASD: The International Journal of Research and Practice, 10, 37-51.

[6] Dalrymple, N. (1992) Some Interpersonal Social Skill Objectives and Teaching Strategies for People with ASD, Revised, Social Series. Indiana Resource Center for ASD; 1994. 1-34.

[7] McAlonan, G.M., Cheung, V., Cheung, C., Suckling, J., Lam, G., Tai, K.S., Yip, L., Declan, G.M. and Siew, E.C. (2005) Mapping the Brain in ASD. A Voxel-Based MRI Study of Volumetric Differences and Intercorrelations in ASD. Brain, 128, 268-176. https://doi.org/10.1093/brain/awh332

[8] Pennington, B.F. and Ozonoff, S. (1996) Executive Functions and Developmental Psychopathology. Journal of Child Psychology and Psychiatry, 37, 51-87. https://doi.org/10.1111/j.1469-7610.1996.tb01380.x

[9] Lopez, B.R., Lincoln, A.J., Ozonoff, S. and Lai, Z. (2005) Examining the Relationship between Executive Functions and Restricted, Repetitive Symptoms of Autistic Disorder. Journal of ASD and Developmental Disorders, 35, 445-460.

https://doi.org/10.1007/s10803-005-5035-x

[10] American Psychiatric Association (2000) Diagnostic and Statistical Manual of Mental Disorders. 4th Edition, Text Revision, American Psychiatric Association, Washington DC.

[11] Lord, C., Rutter, M., Goode, S., Heemsbergen, J., Mawhood, L. and Schopler, E. (1989) Autism Diagnostic Observation Schedule: A Standardized Observation of Communicative and Social Behavior.

[12] Gilliam, J. (2006) Gars-2: Gilliam Autism Rating Scale. 2nd Edition. Pro-ED, Austin.

[13] Wechsler, D., Kaplan, E., Fein, D., Kramer, J., Morris, R., Delis, D. and Maelender, A. (2003) Wechsler Intelligence Scale for Children: Fourth Edition (WISC-IV) [Assessment Instrument]. Pearson, San Antonio.

[14] Gilotty, L., Kenworthy, L., Sirian, L., Black, D.O. and Wagner, A.E. (2002) Adaptive Skills and Executive Function in ASD Spectrum Disorders. Clinical Neuropsychology, 8, 241-248.

[15] Delis, D.C., Kaplan, E. and Kramer, J.H. (2001) Delis-Kaplan Executive Function System (D-KEFS). The Psychological Corporation, San Antonio.

[16] Baron, R. and Parker, J. (2000) Emotional Quotient Inventory: Youth Version (Bar-On EQi: YV). SAGE, San Diego. 